\author{
CONVEX AND FRACTAL GEOMETRY \\ BANACH CENTER PUBLICATIONS, VOLUME 84 \\ INSTITUTE OF MATHEMATICS \\ POLISH ACADEMY OF SCIENCES \\ WARSZAWA 2009
}

\title{
THE DETERMINATION OF CONVEX BODIES FROM THE SIZE AND SHAPE OF THEIR PROJECTIONS AND SECTIONS
}

\author{
PAUL GOODEY \\ Department of Mathematics, University of Oklahoma \\ Norman, Oklahoma 73019, USA \\ E-mail: pgoodey@ou.edu
}

\begin{abstract}
We survey results concerning the extent to which information about a convex body's projections or sections determine that body. We will see that, if the body is known to be centrally symmetric, then it is determined by the size of its projections. However, without the symmetry condition, knowledge of the average shape of projections or sections often determines the body. Rather surprisingly, the dimension of the projections or sections plays a key role and exceptional cases do occur but appear to be sporadic. In a rather different direction, we will see that combining information about the size of projections or sections with other information such as Steiner points or centres of gravity also leads to complete determination of the original body.
\end{abstract}

1. Introduction. This article is a survey of results concerning the determination of a convex or star-shaped body from data associated with its projections or sections. Perhaps the result which motivates almost everything presented here is Aleksandrov's Projection Theorem [1]. In its most basic form it states that if $K_{1}$ and $K_{2}$ are full dimensional centrally symmetric convex bodies in $\mathbb{E}^{d}$ with $V_{d-1}\left(K_{1} \mid u^{\perp}\right)=V_{d-1}\left(K_{2} \mid u^{\perp}\right)$ for all $u \in$ $S^{d-1}$, then $K_{1}$ and $K_{2}$ are the same up to a translation. Thus centrally symmetric bodies with projections of equal volumes are equal up to translation; a full discussion of this and related results can be found in Gardner's book [10].

Not surprisingly, there have been many developments since the time of Aleksandrov's work. Here, we will focus primarily on more recent results similar in nature to Aleksandrov's and especially those which attempt to avoid the symmetry hypothesis. We will also try to emphasize the occasional, and somewhat surprising, interaction between sections and projections.

2000 Mathematics Subject Classification: Primary 52A20; Secondary 52A22, 52A38.

Key words and phrases: convex bodies, projections and sections, Fourier transforms, spherical harmonics.

The paper is in final form and no version of it will be published elsewhere. 
Although our interest is in the geometrical aspects, we will also review the analytical background, since it is here that we often find the basis for the connections between the various results. The analytical aspects come primarily in the form of harmonic analysis and the study of integral operators, on spheres or Grassmannians, which intertwine the action of a rotation group.

There are many topics which are close to our interests but will not be followed here. The first of these is stability. Many of the results we mention have stability versions. For example, Bourgain and Lindenstrauss [3] gave a stability version of Aleksandrov's result. This makes precise the statement that, if $V_{d-1}\left(K_{1} \mid u^{\perp}\right)$ and $V_{d-1}\left(K_{2} \mid u^{\perp}\right)$ are close for all $u \in S^{d-1}$ then $K_{1}$ and $K_{2}$ are almost translates of each other. Another is the issue of inequalities. For example, one might ask what conclusions can be drawn from the hypothesis $V_{d-1}\left(K_{1} \mid u^{\perp}\right) \geq V_{d-1}\left(K_{2} \mid u^{\perp}\right)$ for all $u \in S^{d-1}$. In fact, this is very closely related to Shephard's problem [39]. An analogous problem, for central sections of a star-shaped body, is called the Busemann-Petty problem. In fact, investigations of these two problems led to many of the techniques that will be discussed here. However, a detailed discussion would take us too far from our main topic. The interesting history and resolution of these problems can be found in the books of Gardner [10], Koldobsky [27] and Schneider [36].

In $\S 2$, we will recall the intrinsic volumes which will provide our standard notion of size. There, we will also put the Cauchy-Kubota and Crofton formulas into the context of Radon transforms between Grassmannians. In $\S 3$, we will review some of the standard results which have been established for centrally symmetric objects. $\S 4$ will comprise some of the spherical harmonic analysis aspects which underly the results, emphasizing the aspects which correspond to the symmetry hypotheses in the geometric applications. In $\S 5$ we will move to the study of shapes of sections and projections. In fact, the emphasis will be on average shapes of sections and projections. Here, again, we will encounter unexpected interactions between sections and projections. We will also find some results which fail in certain exceptional dimensions. In $\S 6$, we will see how the work of Groemer [24] and [25] can be put into the general setting of directed data. Finally, $\S 7$ will address some of the possible pairings of data regarding sections and projections that can be used to determine a body uniquely.

The author thanks the referee for numerous suggestions regarding an earlier version of this work.

2. Sizes of sections and projections. Our notion of size is based on the intrinsic volumes $V_{0}, \ldots, V_{d}$. They may be defined using Steiner's formula

$$
V_{d}\left(K+r B^{d}\right)=\sum_{j=0}^{d} \kappa_{d-j} V_{j}(K) r^{d-j},
$$

which expresses the volume of the set of points distance at most $r \geq 0$ from the convex body $K \subset \mathbb{E}^{d}$ as a polynomial in $r$. The number $\kappa_{i}$ appearing in the coefficients denotes the volume of the $i$-dimensional unit ball. We will use Schneider's book [36] as a standard reference for these and other classical notions in convexity. As we shall see, the $j$-th intrinsic volume $V_{j}$ measures $j$-dimensional size $(0 \leq j \leq d)$ in a fairly natural way. 
The fundamental nature of intrinsic volumes lies in the observation that any continuous, motion invariant valuation defined on convex bodies is a linear combination of intrinsic volumes. The Cauchy-Kubota formulae provide a very appealing description of the $j$-th intrinsic volume of a body $K \subset \mathbb{E}^{d}$ in terms of the volumes of its orthogonal projections $K \mid L$ onto members $L$ of the Grassmann manifold $G(d, j)$ of $j$-dimensional subspaces of $\mathbb{E}^{d}$. For a convex body $K \subset \mathbb{E}^{d}$ and $0 \leq j \leq d$, they assert that

$$
V_{j}(K)=c_{d, j} \int_{G(d, j)} V_{j}(K \mid L) d L .
$$

Here, and in the following, symbols such as $c_{d, j}$ denote positive constants dependent only on the dimensions $d$ and $j$. This describes the $j$-th intrinsic volume of $K$ as an average of the $j$-dimensional volumes of the orthogonal projections of $K$ onto $j$-dimensional subspaces of $\mathbb{E}^{d}$, the integration is with respect to the unique rotation invariant probability measure on $G(d, j)$. The Crofton formulae, on the other hand, express intrinsic volumes as average sizes of sections. For a convex $K \subset \mathbb{E}^{d}$ and $0 \leq j \leq k \leq d$, they state

$$
V_{d+j-k}(K)=c_{d, j, k} \int_{A(d, k)} V_{j}(K \cap E) d E ;
$$

here, $A(d, k)$ denotes the affine Grassmannian of $k$-flats in $\mathbb{E}^{d}$ and the integration is with respect to a suitably normalized motion invariant measure on $A(d, k)$. A convenient reference for both the Cauchy-Kubota and the Crofton formulae is the book [38] by Schneider and Weil. The combination of these two formulae provides us with our first example of an unexpected inter-relationship between average sizes of projections and sections.

It is convenient to express some of our results in the language of Radon transforms. For $1 \leq j<k \leq d-1$, these are mappings

$$
R_{j, k}: C(G(d, j)) \rightarrow C(G(d, k))
$$

defined by

$$
\left(R_{j, k} f\right)(L)=\int_{\substack{M \in G(d, j) \\ M \subset L}} f(M) d M,
$$

where the integration is with respect to the unique invariant probability measure on the $j$-dimensional subspaces of $L \in G(d, k)$. In this terminology, the Cauchy-Kubota formulae can be reformulated as stating that

$$
\left(R_{j, k} V_{j}(K \mid \cdot)\right)(L)=c_{d, j, k} V_{j}(K \mid L),
$$

for $1 \leq j<k \leq d-1$ and $L \in G(d, k)$.

Of course, we can also study the Radon transforms

$$
R_{k, j}: C(G(d, k)) \rightarrow C(G(d, j))
$$

defined by

$$
\left(R_{k, j} f\right)(L)=\int_{\substack{M \in G(d, k) \\ M \supset L}} f(M) d M
$$


for $1 \leq j<k \leq d-1$. Applying this transform to intrinsic volumes yields another incidence of the relationship between projections and sections. We have

$$
\left(R_{k, j} V_{k}(K \mid \cdot)\right)(L)=c_{d, j, k} \int_{L} V_{k-j}\left(K \cap\left(L^{\perp}+x\right)\right) d x
$$

where the integration is with respect to Lebesgue measure in $L$; see Goodey, Schneider and Weil [17]. In fact, this result can be used to prove the Crofton formula (3). We note that $R_{j, k}$, for $j<k$ is injective precisely when $j+k \leq d$, whereas, for $j>k$, it is injective precisely when $j+k \geq d$.

3. Centrally symmetric bodies. It is a classical result of Aleksandrov [1] that a centrally symmetric convex body is determined, up to translation, by the size of its projections. To be precise, he showed that if $K_{1}$ and $K_{2}$ are centrally symmetric $d$ dimensional convex bodies in $\mathbb{E}^{d}$ then

$$
V_{d-1}\left(K_{1} \mid u^{\perp}\right)=V_{d-1}\left(K_{2} \mid u^{\perp}\right) \quad \text { for all } u \in S^{d-1} \Leftrightarrow K_{1}=K_{2}+t \text {, for some } t \in \mathbb{E}^{d} .
$$

We note that this result shows that, in the presence of central symmetry, equality of the sizes of projections is equivalent to equality of the shape (and orientation) of the bodies. The result can be proved using the injectivity properties of the cosine transform $C: C\left(S^{d-1}\right) \rightarrow C\left(S^{d-1}\right)$ defined by

$$
(C f)(u)=\int_{S^{d-1}}|\langle u, v\rangle| f(v) d v \quad \text { for } u \in S^{d-1}
$$

here $\langle x, y\rangle$ denotes the scalar product of the vectors $x, y \in \mathbb{E}^{d}$. As we will explain in the next section, the kernel of $C$ comprises the odd functions. Thus, if $f$ and $g$ are even with $C f=C g$ then $f=g$. It is clear that $C$ can be extended to measures $\mu$ on $S^{d-1}$ by

$$
(C \mu)(u)=\int_{S^{d-1}}|\langle u, v\rangle| \mu(d v) \quad \text { for } u \in S^{d-1} .
$$

Thus, measures are mapped to (continuous) functions and Fubini's theorem shows that the above definition is consistent with the standard extension from functions to measures given by

$$
\int_{S^{d-1}} f(u)(C \mu)(d u)=\int_{S^{d-1}}(C f)(v) \mu(d v) \quad \text { for all } f \in C\left(S^{d-1}\right) .
$$

The image of, $C_{e}\left(S^{d-1}\right)$, the even continuous functions on the sphere under $C$ is dense in $C_{e}\left(S^{d-1}\right)$ and so, if $\mu$ and $\nu$ are even measures with $C \mu=C \nu$ then $\mu=\nu$. Aleksandrov's result makes use of the notion of surface area measure, see [36]. These can be viewed as local versions of the intrinsic volumes defined in (1). Instead of measuring the set of all points within a certain distance of the body $K$, we only measure certain parts of this set. To describe this we recall that, for any $x \in \mathbb{E}^{d}$, there is a unique nearest point $p(K, x)$ of $K$ to $x$. For any Borel set $\omega \subset S^{d-1}$ and any $r>0$, we put

$$
U_{r}(K, \omega)=\left\{x \in\left(K+r B^{d}\right) \backslash K:(x-p(K, x)) /\|x-p(K, x)\| \in \omega\right\} .
$$


In analogy with (1), we find that the volume of this set is also a polynomial in $r$. We write this as

$$
V_{d}\left(U_{r}(K, \omega)\right)=\frac{1}{d} \sum_{j=0}^{d-1} r^{d-j}\left(\begin{array}{l}
d \\
j
\end{array}\right) S_{j}(K ; \omega) .
$$

For each $j=1, \ldots, d-1, S_{j}(K ; \cdot)$ is a Borel measure on $S^{d-1}$ which determines $K$ up to translation if $\operatorname{dim} K \geq j+1$. It is called the $j$-th surface area measure of $K$. Comparing (1) and (4), one easily sees that the total measures are multiples of the intrinsic volumes, to be precise,

$$
\frac{1}{d}\left(\begin{array}{l}
d \\
j
\end{array}\right) S_{j}\left(K ; S^{d-1}\right)=\kappa_{d-j} V_{j}(K)
$$

It is also easy to deduce that $K$ is centrally symmetric if and only if $S_{j}(K ; \cdot)$ is an even measure (assuming $\operatorname{dim} K \geq j+1$ ).

The relevance of these measures to Aleksandrov's result comes from the representation

$$
V_{d-1}\left(K \mid u^{\perp}\right)=\frac{1}{2} \int_{S^{d-1}}|\langle u, v\rangle| S_{d-1}(K ; d v)=\frac{1}{2}\left(C S_{d-1}(K ; \cdot)\right)(u) \quad\left(u \in S^{d-1}\right) .
$$

Thus $V_{d-1}\left(K_{1} \mid u^{\perp}\right)=V_{d-1}\left(K_{2} \mid u^{\perp}\right)$ for all $u \in S^{d-1}$, if and only if $S_{d-1}\left(K_{1} ; \cdot\right)$ and $S_{d-1}\left(K_{2} ; \cdot\right)$ have the same cosine transform. In the presence of central symmetry, we now see that the measures must be the same and therefore the bodies are translations of one another. In fact, the same argument gives a stronger result. In general, for $1 \leq j \leq d-1$, we have

$$
V_{j}\left(K_{1} \mid u^{\perp}\right)=V_{j}\left(K_{2} \mid u^{\perp}\right) \Leftrightarrow C S_{j}\left(K_{1} ; \cdot\right)=C S_{j}\left(K_{2} ; \cdot\right) .
$$

Thus, if $K_{1}, K_{2} \subset \mathbb{E}^{d}$ are centrally symmetric convex bodies of dimension at least $j+1$ and if, for some $1 \leq j \leq d-1, V_{j}\left(K_{1} \mid u^{\perp}\right)=V_{j}\left(K_{2} \mid u^{\perp}\right)$ for all $u \in S^{d-1}$ then $K_{1}$ and $K_{2}$ are translates of one another. The combination of this version of Aleksandrov's result with the Cauchy-Kubota formulas (2) shows that, for any $1 \leq j \leq d-1$,

$$
V_{j}\left(K_{1} \mid E\right)=V_{j}\left(K_{2} \mid E\right) \quad \text { for all } E \in G(d, j) \Leftrightarrow K_{1}=K_{2}+t \text {, for some } t \in \mathbb{E}^{d},
$$

again assuming that $K_{1}$ and $K_{2}$ are centrally symmetric and of dimension at least $j+1$.

It is natural, therefore, to ask for analogous results for sets which are not centrally symmetric. In order to discuss this further, we define the $i$-th projection class $(1 \leq i \leq$ $d-1$ ) of a convex body $K \subset \mathbb{E}^{d}$ to be the set of all convex bodies $K^{\prime} \subset \mathbb{E}^{d}$ such that

$$
V_{i}\left(K^{\prime} \mid E\right)=V_{i}(K \mid E) \quad \text { for all } E \in G(d, i) .
$$

In this context, Aleksandrov's result can be interpreted as saying that there is at most one (up to translation) centrally symmetric body in any projection class.

The cases $i=1, d-1$ deserve special mention since, here, there are certain additivity properties. The first of these arises from the fact that $V_{1}\left(\lambda K_{1}+\mu K_{2}\right)=\lambda V_{1}\left(K_{1}\right)+$ $\mu V_{1}\left(K_{2}\right)$ for any convex bodies $K_{1}, K_{2}$ and any $\lambda, \mu \geq 0$. In particular

$$
V_{1}(K)=V_{1}\left(\frac{1}{2} K+\frac{1}{2}(-K)\right)
$$

and so every first projection class contains a centrally symmetric member. The same observation is true for $(d-1)$-st projection classes. However, this observation relies on 
the notion of Blaschke summation $K_{1} \# K_{2}$ of convex bodies $K_{1}, K_{2}$. Minkowski showed that a measure $\mu$ on $S^{d-1}$ is the $(d-1)$-st surface area measure of a full dimensional convex body precisely when its centroid is the origin and it is not supported on a great subsphere; see [36], for example. It follows that, if $K_{1}, K_{2}$ are full dimensional then there is a convex body $K_{1} \# K_{2}$, defined, up to translation, by

$$
S_{d-1}\left(K_{1} \# K_{2} ; \cdot\right)=S_{d-1}\left(K_{1} ; \cdot\right)+S_{d-1}\left(K_{2} ; \cdot\right) .
$$

From this, one can deduce that the $(d-1)$-st projection class of $K$ always contains the centrally symmetric body $(1 / 2)^{1 /(d-1)}(K \#(-K))$.

However, it was shown in [17] that, for $1<i<d-1$, there are non-symmetric (and symmetric) bodies, $K$ such that the $i$-th projection class contains only $K$ and $-K$, the reflection of $K$ in the origin. Chakerian and Lutwak [5] proved that, if an $i$-th projection class contains a centrally symmetric body, that body, uniquely, has maximum volume amongst all bodies in the projection class. Using this and the Cauchy-Kubota formulas, it follows that a centrally symmetric body is determined (amongst all convex bodies) by any two of its projection classes. Campi [4], on the other hand, showed that there are convex bodies that are not determined even by knowledge of all their projection functions.

The analogue, for sections, of Aleksandrov's result states that two centred star-shaped bodies having central sections of equal volumes are the same. To be precise, if the bodies are centred at the origin then

$$
V_{d-1}\left(K_{1} \cap u^{\perp}\right)=V_{d-1}\left(K_{2} \cap u^{\perp}\right) \quad \text { for all } u \in S^{d-1} \Leftrightarrow K_{1}=K_{2} .
$$

In terms of the radial function $r_{K}$ of a star-shaped body this states that

$$
\int_{S^{d-1} \cap u^{\perp}} r_{K_{1}}^{d-1}(v) d v=\int_{S^{d-1} \cap u^{\perp}} r_{K_{2}}^{d-1}(v) d v \quad \text { for all } u \in S^{d-1} \Leftrightarrow K_{1}=K_{2} .
$$

The proof makes use of the spherical Radon transform (which is a variant of $R_{1, d-1}$ ) $R: C\left(S^{d-1}\right) \rightarrow C\left(S^{d-1}\right)$ given by

$$
(R f)(u)=\int_{S^{d-1} \cap u^{\perp}} f(v) d v \quad\left(u \in S^{d-1}\right),
$$

and the fact that it is injective on even functions; see [23], for example. More generally, if $K$ is star-shaped about $o$ and $i>0$, the $i$-th chordal function of $K$ is the function $r_{K}^{i}(u)+r_{K}^{i}(-u)\left(u \in S^{d-1}\right)$. Two star-shaped bodies having equal $i$-th chordal functions are not necessarily equal, unless they are centrally symmetric. However, Gardner and Volčic [11] show that if $i \neq j$ and $K_{1}$ and $K_{2}$ have equal $i$-th and $j$-th chordal functions then $K_{1}=K_{2}$ if one of them is centrally symmetric. In a different direction Falconer [8] and Gardner [12] independently show that if $p_{1} \neq p_{2}$ are interior points of a convex body $K \subset \mathbb{E}^{d}$ then $K$ is determined by knowledge of the $(d-1)$-dimensional volumes of the sections of $K$ through $p_{1}$ and $p_{2}$. This result has recently by generalized by Koldobsky and Shane [29]. They consider the volumes of sections through a single interior point $p$. They show that, if $-1<q<d-1$ is not an integer then the body is uniquely determined by knowledge of the derivative, of order $q$, of parallel section functions at $p$. In the case $0 \leq q<d-1$ is an integer then derivatives of order $q$ at two different interior points are required (except when $q=d-2$ is odd). As has been noted by Falconer [8] and others, 
a convex body is determined, up to reflection in $p$, by the volumes of sections through the boundary point $p$; this can be deduced from the injectivity of the spherical Radon transform, mentioned above.

Further results regarding sections can be found in [10].

4. Analytical aspects. We have already alluded to the roles played by the cosine transform and the spherical Radon transform. Here we will give a brief outline of the harmonic analysis underlying these and subsequent comments. We assume that the mapping $I: C^{\infty}\left(S^{d-1}\right) \rightarrow C^{\infty}\left(S^{d-1}\right)$ is linear and intertwines the group action of $S O(d)$ in the sense that $(I f)_{\rho}=I f_{\rho}$ for each $f \in C^{\infty}\left(S^{d-1}\right)$ and each $\rho \in S O(d)$; here, $f_{\rho}$ is defined by $f_{\rho}(u)=f\left(\rho^{-1} u\right)$ for $u \in S^{d-1}$. The spaces $\mathcal{H}_{n}^{d}(n=0,1, \ldots)$ of $d$-dimensional spherical harmonics of degree $n$ are the irreducible invariant subspaces of $C^{\infty}\left(S^{d-1}\right)$. It therefore follows from Schur's Lemma that $I$ acts as a multiple of the identity on any one of these spaces. Thus, for each $n$, there are numbers (the multipliers, or eigenvalues, of I) $\alpha_{d, n}$ such that $I f=\alpha_{d, n} f$ if $f \in \mathcal{H}_{n}^{d}$.

The archetypal example of such an intertwining operator is the spherical Laplacian $\Delta_{0}$. For a function $f \in C^{\infty}\left(S^{d-1}\right)$, we denote by $F$ the homogeneous degree 0 extension of $f$ to $\mathbb{E}^{d}$. Then $\Delta_{0} f$ is the restriction of $\Delta F$ to $S^{d-1}$. The name spherical harmonic arises from the fact that $\mathcal{H}_{n}^{d}$ is the restriction to the sphere of the set of homogeneous degree $n$ harmonic polynomials on $\mathbb{E}^{d}$; see [23], for example. We also have

$$
\Delta_{0} f=-n(n+d-2) f \quad \text { for } f \in \mathcal{H}_{n}^{d} .
$$

If the $\left|\alpha_{d, n}\right|$ are bounded as functions of $n$, the mapping $I$ may be extended to $I$ : $L^{2}\left(S^{d-1}\right) \rightarrow L^{2}\left(S^{d-1}\right)$ and this mapping is injective precisely when $\alpha_{d, n} \neq 0$ for all $n$. Both the spherical Radon and the cosine transforms can be viewed this way and their multipliers are non-zero precisely when $n$ is even. Specifically, we have

$$
R f=(-1)^{n}(d-1) \kappa_{d-1} \frac{1.3 \ldots(2 n-1)}{(d-1)(d+1) \ldots(d+2 n-3)} f \quad \text { for } f \in \mathcal{H}_{2 n}^{d},
$$

$\left(R f=(d-1) \kappa_{d-1} f\right.$ for $\left.f \in \mathcal{H}_{0}^{d}\right)$ and

$$
C f=2(-1)^{n-1} \kappa_{d-1} \frac{1.3 \ldots(2 n-3)}{(d-1)(d+1) \ldots(d+2 n-1)} f \quad \text { for } f \in \mathcal{H}_{2 n}^{d},
$$

$\left(C f=2 \kappa_{d-1} f\right.$ for $f \in \mathcal{H}_{0}^{d}$ and $C f=(2 /(d+1)) \kappa_{d-1} f$ for $\left.f \in \mathcal{H}_{2}^{d}\right)$.

It is easy to see that the odd multipliers are all zero. It follows that these transforms are injective on even functions. This can be re-phrased by saying that the spherical Radon and cosine transforms provide the even information about functions to which they are applied. Schneider [33] used observations such as these to show the denseness of $C\left(C_{e}\left(S^{d-1}\right)\right)$ in $C_{e}\left(S^{d-1}\right)$ mentioned above.

The hemispherical transform $\tau: L^{2}\left(S^{d-1}\right) \rightarrow L^{2}\left(S^{d-1}\right)$, given by

$$
(\tau f)(u)=\int_{S^{d-1} \cap u^{+}} f(v) d v, \quad u \in S^{d-1},
$$


provides another well-known intertwining mapping. Its multipliers vanish for all even $n \neq 0$ and so $\tau f$ retains the odd information about the function $f$. Specifically, we have

$$
\tau f=(-1)^{n} \kappa_{d-1} \frac{1.3 \ldots(2 n-1)}{(d+1)(d+3) \ldots(d+2 n-1)} f \quad \text { for } f \in \mathcal{H}_{2 n+1}^{d} .
$$

In addition, $\tau f=\left(d \kappa_{d} / 2\right) f$ for $f \in \mathcal{H}_{0}^{d}, \tau f=\kappa_{d-1} f$ for $f \in \mathcal{H}_{1}^{d}$ and all the other even multipliers are zero.

The values of the multipliers given above, together with the detailed spherical harmonic analysis associated with them, can be found in Groemer's book [23].

We will often be in the situation where our mapping $I$ can be extended to measures or distributions on the sphere. The injectivity properties of these extensions are also obtained from the multipliers.

Many of the results mentioned already, as well as those in the sequel, are obtained by associating with the geometrical problem an intertwining operator $I$ and ascertaining its injectivity properties from the values of its multipliers or estimates thereof. In the sequel we will find instances when all the multipliers are non-zero and thus we will be able to deduce that the bodies are determined by the corresponding data. In the later sections we will work with pairs of operators with one determining the even part of the function and the other the odd part, such would be the case if the operators were the Radon and hemispherical transforms, for example.

5. Average shape of sections and projections. We recall that any convex body $K \subset \mathbb{E}^{d}$ is uniquely determined by its support function $h(K ; \cdot)$ defined by

$$
h(K, x)=\max _{k \in K}\langle x, k\rangle \quad\left(x \in \mathbb{E}^{d}\right) ;
$$

we will occasionally denote the support function of $K$ by $h_{K}$. Any subadditive, homogeneous degree 1 function on $\mathbb{E}^{d}$ is a support function. Analogously, any star-shaped (about $o$ ) body is determined by its radial function and any positive function in $C\left(S^{d-1}\right)$ is a radial function.

The support function of the projection of a convex body $K$ onto a subspace $E$ of $\mathbb{E}^{d}$ is just the restriction of $h_{K}$ to $E$. Similarly the radial function of a section of a star-shaped (about $o$ ) body $K$ by a subspace $E$ is the restriction of $r_{K}$ to $E$. It follows that if we know the support function of all projections of a convex body then we know the body, and analogous statements hold for sections of star-shaped bodies. We will therefore focus our attention on certain averages of shapes of projections or sections.

For $1 \leq k \leq d-1$ and $K \subset \mathbb{E}^{d}$ convex, we define the body $P_{k}(K)$ by

$$
h\left(P_{k}(K), u\right)=\int_{G(d, k)} h(K \mid L, u) d L .
$$

The characteristic properties of support functions show that this does define a convex body $P_{k}(K)$. It is also reasonable to refer to this as the average of $k$-dimensional projections of $K$. However, it should be noted that, in general, $\operatorname{dim} P_{k}(K)=d$. These bodies were first introduced by Schneider [35]. His interest was in the possibility that $P_{d-1} K=c K$ for some $c>0$; in fact he showed that this only happens when $K$ is a ball. Our interest is in the question as to whether the body $K$ is determined by $P_{k}(K)$. 
It is fairly easy to show that $P_{1}(K)$ only determines the central symmetrand of $K$. Spriestersbach [40] showed that $P_{d-1}(K)$ determines $K$ uniquely. Goodey [12] extended her techniques to show that $P_{k}(K)$ determines $K$ uniquely if $k \geq d / 2$. He also showed that $P_{2}(K)$ determines $K$ in all dimensions except 14, where it does not determine $K$. In joint work with Jiang [15], it was further shown that $P_{3}(K)$ determines $K$ uniquely. However, not much is known about the situation $4 \leq k<d / 2$ except for a striking result of Kiderlen [26] who showed that $K$ is determined by knowledge of $P_{k}(K)$ for any two values of $k$.

An analogous construction, based on sections, was earlier considered by Goodey and Weil [18]. For $1 \leq k \leq d-1$ they introduced the mean section bodies $M_{k}(K)$ defined by

$$
h\left(M_{k}(K), \cdot\right)=\int_{A(d, k)} h(K \cap E, \cdot) d E .
$$

In this case, $M_{1}(K)$ is always a ball with radius determined by the volume of $K$. They showed that $M_{2}(K)$ always determines the body $K$ but rather little is known in the case $2<k \leq d-1$. Goodey [13] showed that, if $K_{1}, K_{2}$ are centrally symmetric bodies with $M_{k}\left(K_{1}\right)=M_{k}\left(K_{2}\right)$ for any $2 \leq k \leq d-1$ then $K_{1}=K_{2}$.

A (possibly) less obvious sectional analogue of $P_{k}(K)$ was discussed by Goodey, Kiderlen and Weil [16]. This construction makes use of $(d-1)$-st surface area measures of convex bodies $K \subset \mathbb{E}^{d}$. Using Minkowski's characterization of these measures they introduced the sets $B_{k}(K)$ defined, up to translation, by

$$
S_{d-1}\left(B_{k}(K) ; \cdot\right)=\int_{G(d, k)} \int_{L^{\perp}} S_{k-1}^{\prime}(K \cap(L+x) ; \cdot) d x d L,
$$

here $S_{k-1}^{\prime}(K \cap(L+x) ; \cdot)$ denotes the surface area measure of $K \cap(L+x)$ calculated in the $k$-dimensional space $L+x$. This can be described as the Blaschke average of $k$-dimensional sections of $K$. It was shown in [16] that $B_{k}$ has exactly the same injectivity properties as $P_{k}$. In fact rather more is true. As described in the previous section, we can define an intertwining integral operator $p_{k}: C\left(S^{d-1}\right) \rightarrow C\left(S^{d-1}\right)$ such that

$$
h\left(P_{k}(K) ; \cdot\right)=p_{k} h(K: \cdot) \quad \text { for each convex } K \subset \mathbb{E}^{d}
$$

and, for the $B_{k}$, an intertwining operator $b_{k}: \mathcal{M}\left(S^{d-1}\right) \rightarrow \mathcal{M}\left(S^{d-1}\right)$ such that

$$
S_{d-1}\left(B_{k}(K) ; \cdot\right)=b_{k} S_{d-1}(K ; \cdot) \quad \text { for each convex } K \subset \mathbb{E}^{d} ;
$$

here $\mathcal{M}\left(S^{d-1}\right)$ denotes the signed measures on the sphere. Use of Radon-Nikodym derivatives allows one to view $C\left(S^{d-1}\right)$ as a subspace of $\mathcal{M}\left(S^{d-1}\right)$. A surprising observation, from [16] is that $p_{k}$ is precisely the restriction of $b_{k}$ to $C\left(S^{d-1}\right)$. In fact, this is perhaps most easily seen by calculating their multipliers. So these apparently disparate geometric constructions are essentially the same from the analytic point of view.

6. Directed section and projection functions. A brief overview of the results described so far shows that average sizes of projections or sections determine the shape of centrally symmetric bodies and that, in many cases, the average shape of projections or sections determines an arbitrary body. Our plan in this and the subsequent section is to survey some results which attempt to bridge the gap by taking different measure- 
ments of the projections or sections and use these to determine a body without symmetry constraints.

Our approach, here, is to use what we will call directional data. This is motivated by the results of Groemer [24], [25]. For $1 \leq j \leq d-1$, we put

$$
\mathbb{H}_{j}^{d}=\left\{L \cap u^{+}: L \in G(d, j), u \in S^{d-1} \cap L\right\},
$$

and refer to it as the manifold of all $j$-dimensional half-spaces of $\mathbb{E}^{d}$. If $K, M$ are starshaped about the origin and $V_{j}(K \cap H)=V_{j}(M \cap H)$ for all $H \in \mathbb{H}_{j}^{d}$ then one can deduce that $K=M$. To see this, note that we clearly have $V_{j}(K \cap L)=V_{j}(M \cap L)$ for all $L \in G(d, j)$. Thus our earlier observations show that the radial functions of $K$ and $M$ have the same even part. On the other hand, for fixed $L \in G(d, j)$ the restrictions of $r_{K}$ and $r_{M}$ to $L$ have the same odd part since they they have the same hemispherical transform. Thus $r_{K}=r_{L}$ in $\mathbb{E}^{d}$. However, it seems that the body $K$ is over-determined by the $\operatorname{map} \mathbb{H}_{j}^{d} \rightarrow \mathbb{R}$ given by $H \mapsto V_{j}(K \cap H)$.

To investigate this further, we put, for $u \in S^{d-1}$ and $1 \leq j \leq d-1$,

$$
\mathbb{H}_{j}^{(u)}=\left\{L \cap u^{+}: u \in L \in G(d, j)\right\},
$$

and refer to it as the set of $j$-dimensional half-spaces with pole $u$. For a body $K$ which is star shaped with respect to the origin, let

$$
s_{j}(K, u)=\int_{\mathbb{H}_{j}^{(u)}} V_{j}(K \cap H) d H .
$$

We will refer to this as the $j$-th average directed section function of $K$. In case $j=1$, it is just the usual radial function. Goodey and Weil [19] considered the extent to which $K$ is determined by its $j$-th average directed section function. These functions are averages of the functions discussed above. They showed that, if $K, M$ are star-shaped bodies in $\mathbb{E}^{d}$ with

$$
s_{j}(K, u)=s_{j}(M, u) \quad \text { for all } u \in S^{d-1}
$$

for some $j$ with $j \leq(d+2) / 2$ or $j>(2 d+1) / 3$, then $K=M$. However, this implication fails for some $j$, for example $j=(2 d+1) / 3$.

The same authors studied an analogous notion for projections. For $1 \leq i<j \leq d-1$ we put

$$
v_{i, j}(K ; L, u)=S_{i}^{\prime}\left(K \mid L ; u^{+}\right) \quad(u \in L \in G(d, j))
$$

and

$$
\bar{v}_{i, j}(K ; u)=\int_{u \in L \in G(d, j)} v_{i, j}(K ; L, u) d L .
$$

Once again, it was shown that the function $v_{i, j}$ (over-) determines convex bodies up to translation, for much the same reasons as above. It is therefore of interest to study the average directed projection functions $\bar{v}_{i, j}$. The cases $i>1$ result in non-linear problems which seem to be difficult to handle in the general setting. However, Goodey and Weil [20] showed that, if $K, M$ are centrally symmetric convex bodies of dimension at least $i+1$ with $\bar{v}_{i, j}(K ; \cdot)=\bar{v}_{i, j}(M ; \cdot)$ then $K$ and $M$ are translates of one another. It was also shown, for centrally symmetric $K$, that $\bar{v}_{1, j}(K ; \cdot)$ is itself a support function, though 
this is not true in general. The main result of [20] is that, for convex bodies $K, M$ of dimension at least $j+1$ with

$$
\bar{v}_{1, j}(K ; u)=\bar{v}_{1, j}(M ; u) \quad \text { for all } u \in S^{d-1}
$$

for some $j$ with $2 \leq j \leq(2 d-3) / 5$ or $(d-2) / 2 \leq j \leq d-1$, we have $K=M$. Again, this implication fails for some $j$, in particular $j=(2 d-3) / 5$. Three dimensional variations of the above results were studied by Groemer [24], [25].

As mentioned in $\S 4$, one proves results, such as those for $\bar{v}_{1, j}$ by studying the multipliers of an associated analytical mapping. In the case of $\bar{v}_{1, j}$, the odd multipliers are given by

$$
\gamma_{d, j, 2 n+1}=\sum_{k=0}^{n-1}(-1)^{n-k+1} \frac{2(n-k)(2 n-2 k+j)}{j-1} \frac{\left(\frac{1}{2}\right)_{n-k}}{\left(\frac{j+1}{2}\right)_{n-k}} c_{2 n+1, k}^{d, j} .
$$

where

$$
c_{n, k}^{d, j}=\frac{(2(n-2 k)+j-2) n !}{(j-2) k !(n-2 k) !} \frac{\left(\frac{d-2}{2}\right)_{n-k}\left(\frac{d-j}{2}\right)_{k}(j-2)_{n-2 k}}{\left(\frac{j}{2}\right)_{n-k}(d-2)_{n}}
$$

here, we have used the Pochhammer symbol

$$
(a)_{n}=a(a+1) \ldots(a+n-1), \quad \text { for } a \in \mathbb{R} ;
$$

we also put $(a)_{0}=1$, for all $a \in \mathbb{R}$. The issue is to show that these multipliers are all non-zero. The fact that the even multipliers are non-zero follows from geometric results concerning centrally symmetric sets. The study of the odd multipliers seems quite formidable. However, we were able to obtain a rather complex recursion formula by applying an algorithm of Zeilberger. We used the Mathematica package $z b . m$ available via http://www.cis. upenn.edu/ wilf/AeqB.html. It contains an implementation of Zeilberger's Fast Algorithm by Paule, Schorn and Riese, see [31]. This recursion formula allowed us to show that the multipliers are non-zero subject to the dimensional hypotheses described above.

The above average directed projection functions made use of surface area measures and thus required $i<j$. This excludes what is geometrically the most interesting case, namely $i=j$. In [21], another directed projection function was studied. For $u \in S^{d-1}$ and $L \in G(d, j)$ with $u \in L$ we put

$$
p_{1, j}(K ; L, u)=\int_{u^{+} \cap L \cap S^{d-1}} h(K ; v) d v
$$

and

$$
\bar{p}_{1, j}(K ; u)=\int_{u \in L \in G(d, j)} p_{1, j}(K ; L, u) d L .
$$


Analytically, this is the same as the average directed section function $s_{j}(K ; \cdot)$. Consequently, if $K, M \subset \mathbb{E}^{d}$ are convex bodies with

$$
\bar{p}_{1, j}(K, u)=\bar{p}_{1, j}(M, u) \quad \text { for all } u \in S^{d-1}
$$

for $j$ with $j \leq(d+2) / 2$ or $j>(2 d+1) / 3$, then $K=M$. However, the above implication fails for some $j$, for example $j=(2 d+1) / 3$.

As we have seen, there are incidences of non-injectivity (or non-determination) in the above results. A closer analysis of the methods behind Zeilberger's algorithm seems to indicate that these exceptional cases correspond to the occurrence of integer points on algebraic curves. For example, in dimension $d=27, \bar{p}_{1, j}$ is not injective for $j=17$. This happens because $(17,27)$ is an integer point on the non-singular cubic curve

$$
15+138 d-133 j-224 j d+104 d^{2}+105 j^{2}+70 j^{2} y+16 d^{3}-35 j^{3}=0 .
$$

If our suspicions, regarding the correspondence between exceptional cases and integer points on singular curves, is correct then the classical results of number theory concerning rational points on non-singular curves show that there are only sporadic pairs $(j, d)$ where determination fails.

7. Data pairs derived from sections and projections. We now turn our attention to results in which pairs of data from sections or projections are used to determine a body. A good example of this can be found in the work of Böröczky and Schneider [2]. They consider a star-shaped body $K$ in $\mathbb{E}^{d}$ and show that if the volumes and centroids of all the central sections of $K$ are known then $K$ is determined uniquely. The original result of this form is due to Schneider [37]. It involves projections and their Steiner points. We recall that the Steiner point $z(K)$ of a convex body $K \subset \mathbb{E}^{d}$ is the only motion covariant, continuous vector valued function satisfying

$$
z(K+L)=z(K)+z(L)
$$

It is defined by

$$
z(K)=\frac{1}{\kappa_{d}} \int_{S^{d-1}} u h(K ; u) d u
$$

Schneider [37] showed that if, for each $u \in S^{d-1}$, we know $V_{1}\left(K \mid u^{\perp}\right)$ and $z\left(K \mid u^{\perp}\right)$ then $K$ is determined uniquely.

Underlying the results of Schneider and those of Böröczky and Schneider is the spherical Radon transform and the fact that it is injective on even functions.

Building on the ideas of Koldobsky, see [27] for example, Goodey, Yaskin and Yaskina [22], find pairings of integral transforms, one injective on even functions and the other on odd functions using Fourier transforms. For $f \in C^{\infty}\left(S^{d-1}\right)$ and $p \in \mathbb{Z}$, we denote by $f_{p}$ the homogeneous degree $-d+p$ extension of $f$ to $\mathbb{E}^{d} \backslash o$. We then restrict the Fourier transform $\hat{f}_{p}$ to $S^{d-1}$. For appropriate $p$, the mapping $f \mapsto \hat{f}_{p}$ provides an injective map 
$I_{p}: C^{\infty}\left(S^{d-1}\right) \rightarrow C^{\infty}\left(S^{d-1}\right)$. In the cases $p=-1,0,1,2$, for example, we have

$$
\begin{aligned}
& \hat{f}_{-1}(\xi)=-\frac{\pi}{2} \int_{S^{n-1}}|\langle u, \xi\rangle| f(u) d u-i \int_{S^{n-1}}\left(1+\Gamma^{\prime}(1)-\ln |\langle u, \xi\rangle|\right)\langle u, \xi\rangle f(u) d u \\
& \hat{f}_{0}(\xi)=\int_{S^{n-1}}\left(\Gamma^{\prime}(1)-\ln |\langle u, \xi\rangle|\right) f(u) d u \\
& \quad+i \pi\left(\frac{1}{2} \int_{S^{n-1}} f(u) d u-\int_{S^{n-1} \cap \xi^{+}} f(u) d u\right) \\
& \hat{f}_{1}(\xi)=\pi \int_{S^{n-1} \cap \xi^{\perp}} f(u) d u-i \int_{-1}^{1} t^{-1}\left(F_{\xi}(t)-F_{\xi}(0)\right) d t \\
& \hat{f}_{2}(\xi)=-\int_{-1}^{1} t^{-2}\left(F_{\xi}(t)-F_{\xi}(0)-t F_{\xi}^{\prime}(0)\right) d t+2 \int_{S^{n-1} \cap \xi^{\perp}} f(u) d u \\
& \quad-i \pi \int_{S^{n-1} \cap \xi^{\perp}}\left\langle\nabla f_{n+1}(u), \xi\right\rangle d u .
\end{aligned}
$$

In the above, $F_{\xi}$ is defined by

$$
F_{\xi}(t)=\left(1-t^{2}\right)^{(n-3) / 2} \int_{S^{n-1} \cap \xi^{\perp}} f\left(t \xi+\sqrt{1-t^{2}} \zeta\right) d \zeta .
$$

In addition, if $p$ is a non-positive even integer and $f \in C^{\infty}\left(S^{n-1}\right)$ is odd,

$$
\hat{f}_{p}(\xi)=-i(-1)^{-p / 2} \frac{\pi}{(-p) !} \int_{S^{n-1} \cap \xi^{+}}|\langle u, \xi\rangle|^{-p} f(u) d u,
$$

whereas, if $p$ is a negative odd integer and $f$ is even,

$$
\hat{f}_{p}(\xi)=(-1)^{(p-1) / 2} \frac{\pi}{2(-p) !} \int_{S^{n-1}}|\langle u, \xi\rangle|^{-p} f(u) d u
$$

For even $f$, many of the transforms above can be found in [27] and the papers referenced there. The real part of $\hat{f}_{-1}$ was established in [28] and used to provide a Fourier transform solution to the Shephard problem [39]. The most significant feature of the imaginary part of $\hat{f}_{0}$ is the hemispherical transform, which plays an important role in some of Schneider's geometrical applications [34] and in Groemer's work [24], [25]. Its real part was calculated by Yaskin and Yaskina in their work on centroid bodies [41]. The real part of $\hat{f}_{1}$ is the spherical Radon transform which has numerous geometric applications, see Gardner [10] and Groemer [23]. The imaginary part of $\hat{f}_{1}$ may be thought of as a reciprocal cosine transform. We will discuss it in some applications below. The imaginary part of $\hat{f}_{2}$ will be used in the discussion of average heights of shadow boundaries of convex bodies. The integral transforms in (6) and (7) appear in the work of Falconer [7], where he discusses the floating body problem of Ulam. They also play a central role in the study of Lévy representations [30]. More recently, they appeared in [14] in the context of processes of flats in $\mathbb{E}^{d}$ and in [32] in the context of Firey projections of convex bodies. 
It is shown in [22] that the mapping $I_{p}: C^{\infty}\left(S^{n-1}\right) \rightarrow C^{\infty}\left(S^{n-1}\right)$ is injective for real $p$ with $-1 \leq p<n$. Indeed this is an intertwining operator with multipliers

$$
\lambda_{n}(d, p)=\frac{2^{p} \pi^{d / 2}(-1)^{n / 2} \Gamma((n+p) / 2)}{\Gamma((d+n-p) / 2)} \quad \text { if }(p, n) \neq(-1,1) \text { or }(0,0) .
$$

The shadow boundary of the convex body $K \subset \mathbb{E}^{d}$ in the direction $\xi \in S^{d-1}$ comprises those points of the boundary of $K$ which project into a boundary point of $K \mid \xi^{\perp}$. It follows that it is also the set of all boundary points of $K$ at which there are support lines of $K$ parallel to $\xi$. It is easy to see that the average width of a shadow boundary is just the mean width of the corresponding projection. The average height of the shadow boundary in direction $\xi$ of a strictly convex body is defined by

$$
H_{K}(\xi)=\frac{1}{(d-1) \kappa_{d-1}} \int_{S^{d-1} \cap \xi^{\perp}}\left\langle\nabla h_{K}(u), \xi\right\rangle d u .
$$

To see why this definition makes sense, we recall that, for a smooth (and therefore strictly convex) body, the point of contact of the support plane with outer normal $u$ is $\nabla h_{K}(u)$. So the integrand measures the height, above $\xi^{\perp}$, of a point of the shadow boundary of $K$ in direction $\xi$. Of course, we can define the average height of the shadow boundary in any direction for which the shadow boundary is sharp. It is a well-known (and deep) result of Ewald, Larman and Rogers [6] that the shadow boundary of an arbitrary convex body is sharp for almost all $\xi \in S^{d-1}$. Thus $H_{K}$ exists, as an $L^{2}$ function, for all convex bodies $K$. In [22], this was used together with the real part of $\hat{f}_{1}$ and the imaginary part of $\hat{f}_{2}$, in the case $f$ is a support function, to prove that a convex body is determined by knowledge of the average widths and average heights of all its shadow boundaries.

Our next application will involve the real and imaginary parts of $\hat{f}_{1}$ in case $f$ is a support function. In the case of support functions $f=h_{K}$, it is possible to evaluate $\hat{f}_{1}$ without further smoothness conditions. This is already clear for the real part of $\hat{f}_{1}$. To deal with the imaginary part, we note that, for $\xi \in S^{d-1}$ and $u \in S^{d-1} \backslash \xi^{\perp}$,

$$
\frac{1}{2} \frac{h_{K}(u)-h_{K}(-u)}{\langle u, \xi\rangle}
$$

is the average of the heights of the intersection of the support planes with outward normals $\pm u$ with the line, $[\xi]$, through the origin parallel to $\xi$. Consequently it makes sense to refer to

$$
S_{K}(\xi)=\frac{1}{2(d-1) \kappa_{d-1}} \int_{-1}^{1} \int_{S^{d-1} \cap\left(\xi^{\perp}+t \xi\right)} \frac{h_{K}(u)-h_{K}(-u)}{\langle u, \xi\rangle} d u d t
$$

as the average height of the intersection of support planes to $K$ with $[\xi]$, assuming this integral exists. We will now explain briefly why the integral (9) exists for all convex bodies $K$ in $\mathbb{E}^{d}$ and $\xi \in S^{d-1}$ without any smoothness conditions. For $t \in(0,1]$ and $\zeta \in \xi^{\perp} \cap S^{d-1}$, we note that the difference quotients

$$
\frac{h_{K}\left(t \xi+\sqrt{1-t^{2}} \zeta\right)-h_{K}(\zeta)}{t}
$$

are increasing with $t$. Their limit, as $t \searrow 0$, is the directional derivative $h_{K}^{\prime}(\zeta, \xi)$, see [36, Theorem 1.7.2] for example. This, in turn, is the support function of the support set $K \cap\left\{x \in \mathbb{E}^{d}:\langle x, \zeta\rangle=h_{K}(\zeta)\right\}$, evaluated at $\xi$. It follows that, for fixed $\zeta$, these difference 
quotients are integrable with respect to $t \in[0,1]$ and that their integral, as a function of $\zeta$, is bounded. Consequently, $S_{K}(\xi)$ exists for all $K \subset \mathbb{E}^{d}$ and all $\xi \in S^{d-1}$.

A first application of these average heights $S_{K}$ is in the spirit of Schneider's original results [34]. A convex body $K$ is centrally symmetric if and only if $S_{K}$ (or $H_{K}$ ) is a linear function. Equivalently, $S_{K}(\xi)=\langle x, \xi\rangle$ (or $H_{K}(\xi)=\langle x, \xi\rangle$ ) if and only if $K$ is centrally symmetric with respect to $x$. Again this result arises from a study of the multipliers associated with the map $f \mapsto \hat{f}_{1}$ (or $f \mapsto \hat{f}_{2}$ ).

Using both the real and imaginary parts of $\hat{f}_{1}$, we deduce that a convex body $K \subset \mathbb{E}^{d}$ is completely determined by knowledge of the integrals

$$
\int_{S^{d-1} \cap \xi^{\perp}} h_{K}(u) d u \text { and } \int_{-1}^{1} \int_{S^{d-1} \cap\left(\xi^{\perp}+t \xi\right)} \frac{h_{K}(u)-h_{K}(-u)}{\langle u, \xi\rangle} d u d t
$$

for all $\xi \in S^{d-1}$. Thus, $K$ is determined by knowledge of the mean widths of all projections onto hyperplanes and of all the average heights $S_{K}(\xi)$.

The work of Schneider [37] and of Schneider and Böröczky [2] involved the determination of a function $f \in C\left(S^{d-1}\right)$ from certain integrals. The integrals they used were the spherical Radon transforms of $f$ and of the products $\langle\xi, \cdot\rangle f$ for each $\xi \in S^{n-1}$. The former integrals determine the even part of $f$ and the latter, the even part of $\langle\xi, \cdot\rangle f$ or, equivalently, the odd part of $f$. The combination of the two provides unique determination of the function $f$. Their results had elegant geometric interpretations and allowed them to show, on the one hand, that convex bodies are determined by the mean width and Steiner points of their projections and, on the other, a star body is determined by the volumes and centroids of its central sections. In fact, the major part of their work was devoted to establishing stability versions of these results for convex bodies. In this context, we note that knowledge of the spherical Radon transform $R f$ of $f \in C\left(S^{d-1}\right)$ is equivalent to knowing

$$
\int_{S^{d-1}} g(u)(R f)(u) d u \quad \text { for all } g \in C_{e}\left(S^{d-1}\right),
$$

or equivalently

$$
\int_{S^{d-1}} g(u) f(u) d u \quad \text { for all } g \in C_{e}\left(S^{d-1}\right) .
$$

Analogously, knowledge of the spherical Radon transforms of all products $\langle\xi, \cdot\rangle f$ is equivalent to knowing

$$
\int_{S^{n-1}} g(u)\langle\xi, u\rangle f(u) d u \quad \text { for all } g \in C_{e}\left(S^{n-1}\right) \text { and all } \xi \in S^{d-1} \text {. }
$$

However, it follows from the formula for $\hat{f}_{-1}$, that the odd part of $f$ is determined by knowledge of

$$
\int_{S^{d-1}}\left(\Gamma^{\prime}(1)-\ln |\langle u, \xi\rangle|\right)\langle u, \xi\rangle f(u) d u \quad \text { for all } \xi \in S^{d-1} .
$$

Unfortunately, we have not yet found any natural geometric interpretation of these integrals when $f$ is a support function. 
Nevertheless, there is a connection between these approaches. We will show that it is sufficient to know, for each $\xi \in S^{d-1}$, the average of the scalar products $\left\langle z\left(K \mid v^{\perp}\right)\right.$, $\left.\xi\right\rangle$ over all $v \in \xi^{\perp}$, in order to determine the odd part of $h_{K}$. This is the average component, in direction $\xi$, of the Steiner points of the projections of $K$ onto hyperplanes parallel to $\xi$. For a support function $f$, this average is (a multiple of)

$$
\int_{S^{d-1} \cap \xi^{\perp}} \int_{S^{d-1} \cap v^{\perp}}\langle w, \xi\rangle f(w) d w d v .
$$

It is easy to see that the mapping $J: C^{\infty}\left(S^{d-1}\right) \rightarrow C^{\infty}\left(S^{d-1}\right)$ given by

$$
(J f)(\xi)=\int_{S^{d-1} \cap \xi^{\perp}} \int_{S^{d-1} \cap v^{\perp}}\langle w, \xi\rangle f(w) d w d v \quad\left(\xi \in S^{d-1}\right)
$$

is intertwining. As explained in $\S 4$, we can therefore deduce that $J$ acts as a multiple, $\mu_{d, n}$, of the identity on the space $\mathcal{H}_{n}^{d}$ of spherical harmonics. In order to determine the value of $\mu_{d, n}$, it suffices to evaluate $J f$ for one specific $f \in \mathcal{H}_{n}^{d}$. To this end, we recall, see [23] for example, that $\mathcal{H}_{n}^{d}$ is spanned by functions of the form $P_{n}^{d}(\langle\cdot, u\rangle),\left(u \in S^{d-1}\right)$, where $P_{n}^{d}$ is the Legendre polynomial of degree $n$ in dimension $d$. Thus, for any $\xi \in S^{d-1}$,

$$
\begin{aligned}
\mu_{d, 2 n+1} & =\mu_{d, 2 n+1} P_{2 n+1}^{d}(\langle\xi, \xi\rangle)=\left(J P_{2 n+1}^{d}(\langle\cdot \xi\rangle)\right)(\xi) \\
& =\int_{S^{d-1} \cap \xi^{\perp}} \int_{S^{d-1} \cap v^{\perp}}\langle w, \xi\rangle P_{2 n+1}^{d}(\langle w, \xi\rangle) d w d v .
\end{aligned}
$$

Using a standard recursion formula for Legendre polynomials together with (5) and (10) gives

$$
\begin{aligned}
\mu_{d, 2 n+1}=\frac{1}{4 n+d} \int_{S^{d-1} \cap \xi^{\perp}} \int_{S^{d-1} \cap v^{\perp}}\langle w, \xi\rangle\left((2 n+d-1) P_{2 n+2}^{d}(\langle w, \xi\rangle)\right. \\
\left.+(2 n+1) P_{2 n}^{d}(\langle w, \xi\rangle)\right) d w d v
\end{aligned}
$$

The combination of (8) and (11), shows that

$$
\lambda_{2 n+1}(d, 0) \lambda_{2 n+1}(d, 2)=\left(\frac{\pi(d-1)}{d+1}\right)^{2} \mu_{d, 2 n+1} .
$$

It therefore follows that, for odd $f \in C^{\infty}\left(S^{d-1}\right)$, we have

$$
I_{0} I_{2} f=\left(\frac{\pi(d-1)}{d+1}\right)^{2} J f .
$$

Consequently, the average component, in direction $\xi$, of the Steiner points of the projections of $K$ onto hyperplanes parallel to $\xi$ is a multiple of the hemispherical transform $\left(\tau H_{K}\right)(\xi)$. It follows that this determines the odd part of $h_{K}$. 


\section{References}

[1] A. D. Aleksandrov, On the theory of mixed volumes of convex bodies II, New inequalities between mixed volumes and their applications, Mat. Sbornik N. S. 2 (1937), 1205-1238 (in Russian).

[2] K. Böröczky and R. Schneider, Stable determination of convex bodies from projections, Studia Sci. Math. Hungar., to appear.

[3] J. Bourgain and J. Lindenstrauss, Projection bodies, in: Geometric Aspects of Functional Analysis, J. Lindenstrauss and V. D. Milman (eds.), Lecture Notes in Mathematics 1317, Springer, Heidelberg, 1988, 250-270.

[4] S. Campi, Reconstructing a convex surface from certain measurements of its projections, Boll. Un. Mat. Ital. (6) 5-B (1986), 945-959.

[5] G. D. Chakerian and E. Lutwak, Bodies with similar projections, Trans. Amer. Math. Soc. 349 (1997), 1811-1820.

[6] G. Ewald, D. Larman and C. A. Rogers, The direction of the line segments and the $r$ dimensional balls on the boundary of a convex body in Euclidean space, Mathematika 17 (1970), 1-20.

[7] K. J. Falconer, Applications of a result on spherical intregration to the theory of convex sets, Amer. Math. Monthly 90 (1983), 690-693.

[8] K. J. Falconer, X-ray problems for point sources, Proc. London Math. Soc. (3) 46 (1983), 241-262.

[9] R. J. Gardner, Symmetrals and X-rays of planar convex bodies, Arch. Math. 41 (1983), 183-189.

[10] R. J. Gardner, Geometric Tomography, 2nd ed., Cambridge University Press, Cambridge, 2006.

[11] R. J. Gardner and A. Volčič, Tomography of convex and star bodies, Adv. Math. 108 (1994), 367-399.

[12] P. Goodey, Minkowski sums of projections of convex bodies, Mathematika (1998), 253-268.

[13] P. Goodey, Radon transforms of projection functions, Math. Proc. Camb. Phil. Soc. 123, 159-168 (1998).

[14] P. Goodey and R. Howard, Processes of flats induced by higher dimensional processes II, Contemp. Math. 113 (1990), 111-119.

[15] P. Goodey and W. Jiang, Minkowski sums of three dimensional projections of convex bodies, Rend. del Circolo Matematico di Palermo Serie II, Suppl. 65 (2000), 105-119.

[16] P. Goodey, M. Kiderlen and W. Weil, Section and projection means of convex bodies, Monats. Math. 126 (1998), 345-367.

[17] P. Goodey, R. Schneider and W. Weil, Projection functions on higher rank Grassmannians, in: Geometric Aspects of Functional Analysis, J. Lindenstrauss and V. Milman (eds.), Operator Theory: Adv. and Appl. 77, Birkhäuser, Basel, 1995, 75-90.

[18] P. Goodey and W. Weil, The determination of convex bodies from the mean of random sections, Math. Proc. Camb. Phil. Soc. 112 (1992), 419-430.

[19] P. Goodey and W. Weil, Average section functions for star-shaped sets, Adv. in Appl. Math. 36 (2006), 70-84.

[20] P. Goodey and W. Weil, Directed projection functions of convex bodies, Monats. Math. 149 (2006), 43-64.

[21] P. Goodey and W. Weil, Determination of convex bodies by directed projection functions, Mathematika 53 (2006), 49-69. 
[22] P. Goodey, V. Yaskin and M. Yaskina, Fourier transforms and the Funk-Hecke theorem in convex geometry, preprint.

[23] H. Groemer, Geometric Applications of Fourier Series and Spherical Harmonics, Cambridge Univ. Press, New York, 1996.

[24] H. Groemer, On the girth of convex bodies, Arch. Math. 69 (1997), 75-81.

[25] H. Groemer, On a spherical integral transform and sections of star bodies, Monats. Math. 126 (1998), 117-124.

[26] M. Kiderlen, Determination of a convex body from Minkowski sums of its projections, J. London Math. Soc. (2) 70, 529-544 (2004).

[27] A. Koldobsky, Fourier Analysis in Convex Geometry, Mathematical Surveys and Monographs, American Mathematical Society, Providence, RI, 2005.

[28] A. Koldobsky, D. Ryabogin and A. Zvavitch, Projections of convex bodies and the Fourier transform, Israel J. Math. 139 (2004), 361-380.

[29] A. Koldobsky and C. Shane The determination of convex bodies from derivatives of section functions, Arch. Math. 88 (2007), 279-288.

[30] P. Lévy, Théorie de l'addition de variables aléatoires, Gauthiers-Villars, Paris, 1937.

[31] P. Paule and M. Schorn, A Mathematica version of Zeilberger's algorithm for proving binomial coefficient identities, J. Symbolic Comput. 20 (1995), 673-698.

[32] D. Ryabogin and A. Zvavitch, The Fourier transform and Firey projections of convex bodies, Indiana Univ. Math. J. 53 (2004), 667-682.

[33] R. Schneider, Zu einem Problem von Shephard über die Projektionen konvexer Körper, Math. Zeit. 101 (1967), 71-82.

[34] R. Schneider, Über eine Integralgleichung in der Theorie der konvexen Körper, Math. Nachr. 44 (1970), 55-75.

[35] R. Schneider, Rekonstruktion eines konvexen Körpers aus seinen Projektionen, Math. Nachr. 79 (1977), 325-329.

[36] R. Schneider, Convex Bodies: the Brunn-Minkowski Theory, Cambridge University Press, Cambridge, 1993.

[37] R. Schneider, Stable determination of convex bodies from projections, Monatsh. Math. 150 (2007), 241-247.

[38] R. Schneider and W. Weil, Integralgeometrie, Teubner, Stuttgart, 2000.

[39] G. C. Shephard, Shadow systems of convex bodies, Israel J. Math. 2 (1964), 229-236.

[40] K. Spriestersbach, Determination of a convex body from the average of projections and stability results, Math. Proc. Camb. Phil. Soc. 123 (1998), 561-569.

[41] V. Yaskin and M. Yaskina, Centroid bodies and comparison of volumes, Indiana University Math. J. 55 (2006), 1175-1194. 